\title{
Radio recombination lines at decametre wavelengths
}

\section{Prospects for the future}

\author{
W. M. Peters ${ }^{1}$, T. J. W. Lazio ${ }^{1,2, \star}$, T. E. Clarke ${ }^{1}$, W. C. Erickson ${ }^{3}$, and N. E. Kassim ${ }^{1}$ \\ 1 Naval Research Laboratory, 4555 Overlook Ave. SW, Washington, DC 20375, USA \\ e-mail: [Wendy.Peters; Tracy.Clarke; Namir.Kassim]@nrl . navy.mil \\ 2 NASA Lunar Science Institute, NASA Ames Research Center, Moffett Field, CA, USA \\ e-mail: Joseph.Lazio@jpl.nasa.gov \\ 3 University of Tasmania, Churchill Ave., Sandy Bay, Tas 7005, Australia
}

Received 1 April 2010 / Accepted 30 August 2010

\begin{abstract}
This paper considers the suitability of a number of emerging and future instruments for the study of radio recombination lines (RRLs) at frequencies below $200 \mathrm{MHz}$. These lines are of interest because they arise only in low-density regions of the ionized interstellar medium and because they may represent a frequency-dependent foreground for next-generation experiments trying to detect H I signals from the Universe's Epoch of Reionization and Dark Ages (so-called "21-cm cosmology" observations). We summarize existing decametre-wavelength observations of RRLs, which have detected only carbon RRLs. We then show that, with reference to an interferometric array, the primary instrumental factor limiting detection and study of the RRLs is the areal filling factor of the array. We consider the first station of the Long Wavelength Array (LWA-1), the LOw Frequency ARray (LOFAR), the low-frequency component of the Square Kilometre Array (SKA-lo), and a future Lunar Radio Array (LRA), all of which are likely to operate at decametre wavelengths. Key advantages that many of these arrays offer include digital signal processing, which should produce more stable and better defined spectral bandpasses; larger frequency tuning ranges; and better angular resolution than that of the previous generation of instruments that have been used in the past for RRL observations. Detecting Galactic carbon RRLs, with optical depths at the level of $10^{-3}$, appears feasible for all of these arrays, with integration times ranging from a few hours to as much as $100 \mathrm{~h}$; at optimal frequencies this would permit a Galactic survey. The SKA-lo and LRA, and the LWA-1 and LOFAR at the lowest frequencies, should have a high enough filling factor to detect lines with much lower optical depths, of order $10^{-4}$ in a few hundred hours. The amount of RRL-hosting gas present in the Galaxy at the high Galactic latitudes likely to be targeted in Epoch of Reionization and Dark Ages H I studies is currently unknown. If present, however, the spectral fluctuations from RRLs could be comparable to or exceed the anticipated HI signals.
\end{abstract}

Key words. line: identification - instrumentation: interferometers - ISM: lines and bands - radio lines: ISM

\section{Introduction}

In the low-density regions of the Galactic interstellar medium (ISM), free electrons can be captured by ions at very high quantum numbers $(n>100)$. As the atom cascades into a series of successively lower ionization states, each transition produces a radio recombination line (RRL, Gordon \& Sorochenko 2002). These Rydberg atoms are large, often having macroscopic dimensions, and are consequently somewhat fragile. As a result, the transitions between high quantum number levels are a sensitive probe of the environments in which the atoms exist, providing diagnostics such as temperature, density, and pressure, and the lines can be used to constrain the size of the regions in which the atoms occur. RRLs also may be a frequency-dependent contaminant in future astrophysical and cosmological observations using the highly redshifted H I $21-\mathrm{cm}$ line from the Epoch of Reionization (EoR) or earlier.

Konovalenko \& Sodin (1980) discovered the first decametre RRL at a frequency of $26.13 \mathrm{MHz}$, using the UTR-2 telescope to observe Cas A. Blake et al. (1980) subsequently identified

^ Current address: Jet Propulsion Laboratory, M/S 138-308, 4800 Oak Grove Dr., Pasadena, CA 91109, USA. the line as the C631 $\alpha$ line. Since then, carbon RRLs have been measured towards Cas A at frequencies from 14 to $1420 \mathrm{MHz}$ (Payne et al. 1994, and references therein). A recent study by Stepkin et al. (2007) measured $\alpha, \beta, \gamma$, and even $\delta$ carbon-line transitions at frequencies near $20 \mathrm{MHz}$. The lines were measured up to $n \sim 1005$, which is the highest $n$-bound state ever detected.

Observationally, the RRLs resulting from high Rydberg transitions typically occur in emission at frequencies above $200 \mathrm{MHz}$ (shortward of $1.5 \mathrm{~m}$ ) and have line widths dominated by Doppler broadening. Below $150 \mathrm{MHz}$, however, the excitation temperature of the atoms approaches the typical gas kinetic temperature and the lines appear in absorption (Payne et al. 1989). In between is a poorly studied transition region, in which the lines may be undetectable.

There are a number of new telescopes, either under construction or in the design phase (Sect. 5), that will be operating at frequencies below about $200 \mathrm{MHz}$, which we shall henceforth consider "decametre wavelengths." This paper considers the impact these emerging instruments may have on RRL studies, specifically the $\alpha$-transition Carbon RRLs. We review aspects of decametric RRLs from the perspective of the Galactic interstellar medium (Sect. 2) and cosmological observations (Sect. 3), then in Sect. 4 we consider the detection of the lines, in Sect. 5 we 
discuss the specifics of various instruments with respect to RRL observations, and, in Sect. 6 we present our conclusions.

\section{RRLs and the galactic ISM}

Pressure and radiation broadening as well as Doppler effects may affect the RRL absorption line profiles, and can be used to derive physical properties of the absorbing cloud(s) (Kantharia et al. 1998). The relative strengths of the $\alpha, \beta$, and $\gamma$ lines, which can be measured simultaneously at different quantum numbers $n$ in the same spectrum, can be used to constrain the physical properties of the absorbing gas further (Erickson et al. 1995). While hydrogen, helium, and carbon lines have been identified in emission, all low-frequency RRLs known to date are high- $n$ carbon transitions.

Using multi-frequency model fits to all existing data on the Cas A sightline and comparing the spatial distribution of the higher frequency lines to that of $\mathrm{HI}$ and molecular clouds on the sightline, Kantharia et al. (1998) concluded that these RRLs most likely occur in diffuse H I clouds in the Perseus arm, which have temperatures of order $75 \mathrm{~K}$. The best fit physical models, based on Cas A sightline data from all frequencies, predict a stronger broadening in the lowest frequency lines than has been actually observed. According to Kantharia et al. (1998), the discrepancies arise, at least in part, from difficulties in accurately detecting and measuring the Lorentzian wings due to the weakness of the lines and issues with accurate spectral baseline removal. Many lines reported in the literature have been erroneously fit with Gaussians (e.g., Sorochenko \& Smirnov 1990), and others were subjected to a baseline removal process which significantly diminished the fitted line strengths and widths (Payne et al. 1994). In order to fully realize the potential of these lines in measuring physical conditions in the ISM, sensitive observations with robust spectral baselines are needed, particularly at the lowest frequencies. With their very high signalto-noise observations, Stepkin et al. (2007) argue that errors in fitting or spectral baseline removal alone cannot account for the line-width discrepancy; however including the known lowfrequency spectral turnover of Cas A (e.g., Helmboldt \& Kassim 2009) lowers the amount of predicted broadening and brings the model and observations into agreement.

Aside from the Cas A sightline, carbon recombination lines in absorption have been observed on a variety of sightlines in the Galaxy (Tables 1 and 2). Most observations have focussed on sightlines towards known bright background sources or passing through gas-rich regions along the inner Galactic plane, but the detection rates have been low. Only two broader surveys for the lines have been published. Erickson et al. (1995) found carbon RRL absorption complexes on roughly 30 sightlines between Galactic longitudes of $340^{\circ}$ and $20^{\circ}$, with Galactic latitudes $|b|<2^{\circ}$. They concluded that there is no evidence for pressure broadening in their data, and that the linewidths reflect Doppler broadening and the presence of many clouds in their $4^{\circ}$ beam. Kantharia \& Anantharamaiah (2001) found RRLs on 9 sightlines, with six of those in the Galactic longitude range $352^{\circ}<\ell<17^{\circ}$. Combining their data with that of Erickson et al. (1995) and information from observations on the same sightlines at higher frequencies, they conclude that the clouds likely have sizes of $2^{\circ}-4^{\circ}$ and occur in photodissociation regions.

\section{RRLs and 21-cm cosmology}

There is growing interest in the use of the 21-cm hyperfine transition of $\mathrm{HI}$ as a cosmological and astrophysical probe of the distant Universe. For comprehensive reviews, see Furlanetto et al. (2006a) and Pritchard \& Loeb (2008). For a portion of the Universe's history $(1100 \lesssim z \lesssim 7$ ), the baryonic content of the intergalactic medium (IGM) is dominated by $\mathrm{H}$, and interactions between the adiabatic expansion of the IGM and various heating sources (e.g., first stars, first black holes, dark matter decay) can produce either an absorption or emission $\mathrm{H}$ I signal relative to the cosmic microwave background (CMB).

At least three distinct epochs have been identified, which have corresponding spectral windows for the redshifted H I signal.

1. The Dark Ages $-100 \lesssim z \lesssim 30(15 \mathrm{MHz} \lesssim v \lessgtr 50 \mathrm{MHz})$. The H I gas is expanding and cooling at a faster rate than the $\mathrm{CMB}$, and collisions within the gas drive the kinetic temperature of the gas below the $\mathrm{CMB}$ temperature, resulting in an absorption feature. This epoch is thought to occur before the first stars form, and, absent any other heating sources (e.g., energy injection by decaying dark matter, Furlanetto et al. 2006b), the evolution of this signal should depend only on cosmological parameters.

2. First star formation $-30 \lesssim z \lesssim 15(50 \mathrm{MHz} \lesssim v \lesssim 90 \mathrm{MHz})$. As the first stars form, they flood the Universe with Lyman- $\alpha$ photons. These strongly couple the kinetic and spin temperatures of $\mathrm{HI}$ and produce a second, deep absorption feature. Detection of this signal would constrain both the epoch and luminosity function of the first stars.

3. Epoch of Reionization (EoR) $-15 \lesssim z \lesssim 7(90 \mathrm{MHz} \lesssim v \lessgtr$ $200 \mathrm{MHz}$ ). As stars and the first black holes heat the gas, its temperature rises above the CMB temperature. The absorption feature turns into an emission feature, which persists until reionization completes and the signal cuts off. Detection of this feature would constrain the duration of the EoR and the luminosity function(s) of ionizing sources present.

Estimates of the strength of these H I signals is both epoch and model dependent but are in the range $10-100 \mathrm{mK}$.

One of the key challenges to detecting any of these signals are the various foregrounds. These foregrounds include radio frequency interference (RFI) from terrestrial radio transmitters, ionospheric phase corruptions, the Galactic synchrotron emission, and emission from extragalactic sources. The various spectral windows for these $\mathrm{H}$ I signals also include frequencies where RRLs have been observed on Galactic plane sightlines. It is currently unknown at what strength RRLs might exist on sightlines at the high Galactic latitudes where most of the cosmological studies will be made.

A standard assumption for the removal of astrophysical foregrounds (Galactic synchrotron, extragalactic sources) is that their spectra are smooth (Harker et al. 2009). While 21-cm cosmological observations will clearly be conducted at high Galactic latitudes, in order to minimize the contribution of the Galactic synchrotron emission, even residual effects from RRLs might still vitiate the measurements.

As an illustration of the potential impact of RRLs on these experiments, consider a set of RRLs having an optical depth $\tau \sim 5 \times 10^{-4}$, characteristic of what has been observed to date (Table 1). We assume no continuum opacity, as is reasonable for observations at frequencies $v \gtrsim 10 \mathrm{MHz}$ at high Galactic latitudes. The continuum temperature $T_{\mathrm{c}}$ will be the combination of the Galactic synchrotron emission and the extragalactic background but is likely to be dominated by the former (Bridle 1967). The temperature in the line is then $\left(T_{\mathrm{c}}+T\right) \tau$, for a temperature $T$ of the gas hosting the RRLs and employing the usual expansion 
W. M. Peters et al.: Radio recombination lines at decametre wavelengths

Table 1. Known Galactic Carbon $\alpha$ RRL absorption lines ${ }^{a}$.

\begin{tabular}{|c|c|c|c|c|c|c|}
\hline Sightline & $\begin{array}{c}v \\
(\mathrm{MHz})\end{array}$ & $n$ & $\begin{array}{c}\tau \\
\left(\times 10^{-3}\right)\end{array}$ & $\begin{array}{c}V_{\mathrm{LSR}} \\
\left(\mathrm{km} \mathrm{s}^{-1}\right)\end{array}$ & $\begin{array}{c}\Delta v \\
\left(\mathrm{~km} \mathrm{~s}^{-1}\right)\end{array}$ & Reference \\
\hline \multirow{3}{*}{$\overline{\mathrm{G} 0+0}$} & 34.5 & 575 & 1.16 & $5.3 \pm 0.8$ & $20.5 \pm 1.1$ & 5 \\
\hline & 75 & 443 & $0.57 \pm 0.04$ & $-1.0 \pm 0.5$ & $14.8 \pm 1.2$ & APE88 \\
\hline & 76 & 441 & 0.73 & -1 & 24 & 1 \\
\hline $\mathrm{G} 0-4^{b}$ & 76 & 441 & 0.33 & -6 & 13 & 1 \\
\hline G0-2 & 76 & 441 & 0.57 & -10 & 30 & 1 \\
\hline \multirow[t]{2}{*}{$\mathrm{G} 0+0$} & 34.5 & 575 & 1.16 & $5.3 \pm 0.8$ & $20.5 \pm 1.1$ & 5 \\
\hline & 76 & 441 & 0.73 & -1 & 24 & 1 \\
\hline $\mathrm{G} 0+2$ & 76 & 441 & 0.70 & -2 & 26 & 1 \\
\hline $\mathrm{G} 0+4$ & 76 & 441 & 0.68 & -2 & 31 & 1 \\
\hline $\mathrm{G} 2-3.5^{b}$ & 76 & 441 & 0.52 & 7 & 5 & 1 \\
\hline G2-2 & 76 & 441 & 0.97 & 5 & 9 & 1 \\
\hline $\mathrm{G} 2+0$ & 76 & 441 & 0.90 & 2 & 25 & 1 \\
\hline $\mathrm{G} 2+2$ & 76 & 441 & 0.61 & 6 & 11 & 1 \\
\hline $\mathrm{G} 3+0$ & 76 & 441 & 0.90 & -1 & 14 & 1 \\
\hline $\mathrm{G} 4+0$ & 76 & 441 & 0.54 & 8 & 17 & 1 \\
\hline G5+0 & 34.5 & 575 & 0.74 & $10.2 \pm 1.4$ & $21.2 \pm 2.0$ & 5 \\
\hline G6+0 & 76 & 441 & 0.73 & 9 & 25 & 1 \\
\hline G6.6-0.2 & 76 & 441 & 0.87 & 10 & 28 & 1 \\
\hline $\mathrm{G} 8+0$ & 76 & 441 & 1.09 & 11 & 22 & 1 \\
\hline \multirow[t]{4}{*}{$\mathrm{G} 10+0$} & 34.5 & 575 & 0.81 & $14.3 \pm 1.7$ & $37.5 \pm 2.5$ & 5 \\
\hline & & & 0.93 & $9.6 \pm 1.5$ & $24.9 \pm 2.3$ & 5 \\
\hline & & & 0.47 & $40.2 \pm 2.6$ & $18.4 \pm 3.7$ & 5 \\
\hline & 76 & 441 & 0.72 & 17 & 26 & 1 \\
\hline $\mathrm{G} 12+0.0$ & 76 & 441 & 0.91 & 12 & 20 & 1 \\
\hline G14-2.0 & 76 & 441 & 0.88 & 11 & 32 & 1 \\
\hline \multirow[t]{2}{*}{$\mathrm{G} 14+0$} & 34.5 & 575 & 0.66 & $37.8 \pm 2.7$ & $54.0 \pm 3.8$ & 5 \\
\hline & 76 & 441 & 0.85 & 16 & 25 & 1 \\
\hline $\mathrm{G} 14+2.0$ & 76 & 441 & 0.51 & 21 & 28 & 1 \\
\hline $\mathrm{G} 16+0.0$ & 76 & 441 & 0.76 & 14 & 47 & 1 \\
\hline \multirow[t]{2}{*}{ M16 } & 68 & 456 & $1.8 \pm 0.3$ & $18 \pm 2$ & $16 \pm 3$ & 6 \\
\hline & 80 & 435 & $2.0 \pm 0.3$ & $20 \pm 1$ & $13 \pm 2$ & 6 \\
\hline G16.5+0 & 34.5 & 575 & 0.59 & $26.4 \pm 2$ & $32.8 \pm 2.8$ & 5 \\
\hline G06.9+0.8 & 76 & 441 & 1.07 & 20 & 21 & 1 \\
\hline $\mathrm{G} 18+0$ & 76 & 441 & 0.77 & 15 & $33 \pm 6$ & 1 \\
\hline $\mathrm{G} 20+0^{b}$ & 76 & 441 & 0.59 & 36 & $18 \pm 3$ & 1 \\
\hline $\mathrm{G} 63+0$ & 34.5 & 575 & 0.42 & $36.2 \pm 3.2$ & $46.0 \pm 4.5$ & 5 \\
\hline $\mathrm{G} 75+0$ & 25 & 640 & 1 & 12 & 15 & 4 \\
\hline $\mathrm{G} 75+0$ & 34.5 & 575 & 0.40 & $6.9 \pm 1.8$ & $27.1 \pm 2.6$ & 5 \\
\hline \multirow[t]{2}{*}{ DR 21} & 25 & 640 & $0.7 \pm 0.3$ & $0 \pm 8$ & $42 \pm 12$ & 2 \\
\hline & 34.5 & 575 & 0.74 & $4.5 \pm 1.6$ & $18.8 \pm 2.2$ & 5 \\
\hline \multirow[t]{2}{*}{ S140 } & 25 & 640 & $1 \pm 0.3$ & $-6 \pm 17$ & $96 \pm 24$ & 2 \\
\hline & & & $0.5 \pm 0.3$ & $-36 \pm 17$ & $96 \pm 24$ & 2 \\
\hline NGC 2024 & 25 & 640 & 1 & 10 & 36 & 4 \\
\hline G287.4-0.6 $6^{b}$ & 76 & 441 & 0.48 & -23 & 38 & 1 \\
\hline $\mathrm{G} 312+0$ & 76 & 441 & 0.88 & -53 & 16 & 1 \\
\hline $\mathrm{G} 340+0^{b}$ & 76 & 441 & 0.27 & -39 & 30 & 1 \\
\hline $\mathrm{G} 342+0^{b}$ & 76 & 441 & 0.53 & -39 & 17 & 1 \\
\hline G344+0 & 76 & 441 & 0.65 & -27 & 26 & 1 \\
\hline $\mathrm{G} 346+0^{b}$ & 76 & 441 & 0.20 & -20 & 20 & 1 \\
\hline $\mathrm{G} 348+0$ & 76 & 441 & 0.53 & -12 & 14 & 1 \\
\hline $\mathrm{G} 350+0$ & 76 & 441 & 0.81 & -11 & 14 & 1 \\
\hline G352-2.0 & 87 & 441 & 0.70 & -17 & 23 & 1 \\
\hline \multirow[t]{2}{*}{$\mathrm{G} 352+0$} & 34.5 & 575 & 0.65 & $-1.2 \pm 1.8$ & $34.8 \pm 2.6$ & 5 \\
\hline & 76 & 441 & 1.25 & -10 & 11 & 1 \\
\hline $\mathrm{G} 352+2.0$ & 76 & 441 & 0.83 & -8 & 22 & 1 \\
\hline G354+0 & 76 & 441 & 0.87 & -10 & 26 & 1 \\
\hline G356+0 & 76 & 441 & 0.76 & -4 & 17 & 1 \\
\hline G358-2 & 76 & 441 & 0.53 & -10 & 28 & 1 \\
\hline G358+0 & 76 & 441 & 0.90 & -3 & 24 & 1 \\
\hline $\mathrm{G} 358+2$ & 76 & 441 & 0.80 & 3 & 8 & 1 \\
\hline L1407 & 25 & 640 & 0.7 & -10 & 17 & 3 \\
\hline
\end{tabular}

Notes. ${ }^{(a)}$ This listing excludes lines for the Cas A line of sight. ${ }^{(b)}$ Tentative detection.

References. (1) Erickson et al. (1995); (2) Golynkin \& Konovalenko (1991a); (3) Golynkin \& Konovalenko (1991b); (4) Konovalenko (1984); (5) Kantharia \& Anantharamaiah (2001); (6) Anantharamaiah et al. (1988). 
Table 2. Previous and existing instrument capabilities.

\begin{tabular}{lcccc}
\hline \hline Name & $\begin{array}{c}\text { Frequency } \\
(\mathrm{MHz})\end{array}$ & Resolution & Filling factor & $\begin{array}{c}T_{\text {detect }} \\
(\mathrm{h})\end{array}$ \\
\hline UTR-2 & 26 & $40^{\prime}$ & 1 & 1 \\
Gauribidanur & 34.5 & $21^{\prime} \times 25^{\circ}$ & 0.59 & 1.5 \\
DKR-1000 & 40 & $44^{\prime} \times 1^{\circ}$ & 0.2 & 25 \\
Arecibo & 47 & $80^{\prime}$ & 0.25 & 23 \\
Parkes & 76 & $4^{\circ}$ & 0.25 & 2 \\
\hline
\end{tabular}

Notes. $T_{\text {detect }}$ is the time to detect $(5 \sigma)$ a line of optical depth $10^{-3}$ with an well-matched instrumental bandwidth. The instruments listed are ones from which published RRL results have been obtained. In many cases, characteristics have been taken from the literature, and the instrument itself may not be currently operational.

for small optical depths. We now consider the two cases, $T_{\mathrm{c}} \gg T$ and $T_{\mathrm{c}} \sim T$.

Using $150 \mathrm{MHz}$ as a characteristic frequency for detection of the H I signal from the EoR, the coldest parts of the sky have a temperature $T_{\mathrm{c}} \approx 150 \mathrm{~K}$. In the Galactic plane, the gas hosting the RRLs has $T \sim 100 \mathrm{~K}$ (Sect. 2), and if there is such RRLhosting gas at high Galactic latitudes, then clearly $T \sim T_{\mathrm{c}}$. In this case, spectral fluctuations between RRLs and the continuum of order $\left(T_{\mathrm{c}}+T\right) \tau / T_{\mathrm{c}} \sim 2 \tau$ should be expected. Conversely, if one considers $75 \mathrm{MHz}$ as a characteristic frequency for the $\mathrm{H}$ I signal from the first star formation, the coldest parts of the sky have a temperature $T_{\mathrm{c}} \approx 1000$. Assuming, as before, $T=100 \mathrm{~K}$, spectral fluctuations of order $\tau$ should be expected.

The magnitude of the spectral fluctuations expected from the H I signal varies with the epoch being considered, but a typical range of values is $10^{-5}-10^{-4}$. If there is RRL-hosting gas at high Galactic latitudes, the spectral fluctuations arising from RRLs could be comparable to or exceed that from the desired H I signals.

\section{Detection of RRLs}

We begin by reviewing the observational requirements for detecting RRLs. From the radiometer equation, the sensitivity of an experiment designed to detect RRLs can be expressed as

$\Delta T=\frac{T_{\text {sys }}}{f} \frac{1}{\sqrt{N_{\text {lines }} N_{\text {pol }} \Delta t_{\text {int }} \Delta v}}$

where $\Delta T$ is the rms noise, $T_{\text {sys }}$ is the telescope system temperature, $f$ is the filling factor of the telescope or array, $\Delta t_{\text {int }}$ is the integration time, $\Delta v$ is the frequency resolution, $N_{\text {pol }}$ is the number of polarizations, and $N_{\text {lines }}$ is the number of simultaneous transitions observed that can be folded together.

If the RRL has an optical depth $\tau$, then an " $m$-sigma" detection implies $m \Delta T \leq \tau T_{\text {sys }}$, with a corresponding integration time of

$\Delta t_{\text {int }}=\left(\frac{m}{f \times D F} \frac{T_{\text {sys }}}{\Delta T}\right)^{2} \frac{1}{N_{\text {pol }} N_{\text {lines }} \Delta v}$.

We have introduced the beam dilution factor (dilution factor or DF) of the absorbing signal, which is relevant for the case of observations of lines against a non-discrete background such as the Galactic synchrotron emission. For example, estimates from two surveys suggest that absorption lines seen towards the central Galactic plane probably arise in clouds that subtend between $2^{\circ}$ and $4^{\circ}$ on the sky, but are unlikely to be bigger (Kantharia \& Anantharamaiah 2001). If the beam of the antenna
Table 3. Future telescope capabilities.

\begin{tabular}{lcccc}
\hline \hline Name & $\begin{array}{c}\text { Frequency } \\
(\mathrm{MHz})\end{array}$ & Resolution & Filling factor & $\begin{array}{c}T_{\text {detect }} \\
(\mathrm{h})\end{array}$ \\
\hline LWA-1 & $20-80$ & $9^{\circ}-2^{\circ}$ & $0.9-0.1$ & $\sim 10$ \\
LOFAR-LBA & $30-90$ & $2^{\circ}-0.6^{\circ}$ & $\sim 0.1$ & $\sim 10$ \\
LOFAR-HBA & $110-240$ & $30^{\prime}-15^{\prime}$ & $\sim 0.1$ & $\ldots$ \\
SKA-lo & 150 & $7^{\prime}$ & $\sim 0.1$ & $<10$ \\
LRA & 100 & $10^{\prime}$ & $\sim 0.9$ & $<10$ \\
\hline
\end{tabular}

Notes. Values listed are characteristic or indicative, but none of these telescopes have entered an operational state, so actual performance may vary. For LOFAR-LBA, bandwidths of $48 \mathrm{MHz}$ have been assumed. LOFAR-HBA covers a poorly explored frequency range in which the lines may become undetectable as they transition from emission at higher frequencies to absorption at lower frequencies; the same may also be true of SKA-lo. Particularly for the SKA-lo and LRA, final design parameters are yet to be determined, and these values are indicative.

is substantially larger than $4^{\circ}$, it is likely that the absorption feature will be diluted by unabsorbed background radiation. We estimate DF as the geometric ratio of the cloud and beam sizes, and $\mathrm{DF} \leq 1$. The dilution factor DF enters Eq. (2) as a multiplier of $\Delta T$, so $\Delta t_{\text {int }} \propto \mathrm{DF}^{-2}$. This factor is not important when the background source is discrete and thus fully covered by the absorbing gas, such as in the case of Cas A and other bright Galactic or extragalactic sources.

Most previous RRL observations have been conducted at relatively low angular resolutions (Table 2). As a consequence, a significant uncertainty has been the extent to which different source regions are blended within the telescope beam. One of the advantages of many of the future telescopes (Sect. 5) is that they will have better angular resolution than most of the previous systems used for RRL observations. Thus, we include the beam dilution factor DF as a generic factor to account for a potential mismatch between the angular sizes of the of foreground absorber(s) and the background source(s) in the beam. Indeed, future observations, particularly those at different frequencies and therefore different angular resolutions, may help resolve the extent to which a mismatch has been an issue in previous observations.

The system temperature at low radio frequencies is typically dominated by the synchrotron emission from the Galactic plane itself and thus varies with both position and frequency. As can be seen in Table 1, most of the Galactic lines are very weak, with optical depths $\tau \lesssim 10^{-3}$. Even a $5 \sigma$ detection (i.e., $m=5$, equation 2) therefore implies $\Delta T / T_{\text {sys }} \lesssim 2 \times 10^{-4}$. Any survey for these lines should be targeted to reach at least this sensitivity level.

The lines themselves vary in velocity width from 10 to $100 \mathrm{~km} \mathrm{~s}^{-1}$, suggesting that a velocity resolution of roughly $2 \mathrm{~km} \mathrm{~s}^{-1}(\sim 700 \mathrm{~Hz}$ at $100 \mathrm{MHz})$ is appropriate for blind detection of the narrowest lines. However, even observations targeting the broader lines will benefit from high spectral resolution to aid in the identification and excision of RFI. Because the lines themselves are quite weak, even weak RFI must be carefully removed in these observations.

\section{Prospects for future observations}

A new generation of low frequency instruments have either the opportunity to contribute to Galactic RRL and ISM studies or to be confused by the lines when doing other studies (Table 3). Improvements in both sensitivity and resolution will help further resolve issues of physical cloud parameters such as size, 
W. M. Peters et al.: Radio recombination lines at decametre wavelengths

pressure and temperature, while allowing a more complete census of the ionized carbon gas in the Galaxy. Moreover, in many cases, one of the prime science drivers for the telescope is observations of the cosmological H I signal, so that careful removal of foregrounds, including RRLs, is paramount. We shall consider the performance of these telescopes in turn.

At low frequencies, the system temperature $T_{\text {sys }}$ should be dominated by the Galactic synchrontron emission, and all of the telescopes will have the capability to conduct dual polarization observations $\left(N_{\text {pol }}=2\right)$. Thus, with respect to the performance of these future telescopes, most notably those still in the design phase, Eq. (2) indicates that there are only two other factors under the control of those designing the telescope. One is the filling factor $f$ of the telescope and the other is the number of lines $N_{\text {lines }}$, which is coupled to the total bandwidth that the system can handle.

One feature common to all of these future systems is the signal chain will be largely digital, which should enable significant improvements in the stability and shape of the spectral bandpasses. Further, all of the telescopes will have a significantly enhanced frequency tuning range (with typical ranges of 2:1 or better), which will enable many more lines to be observed. Finally, all of the systems that we will discuss will be dipolebased phased array systems in which the dipoles will be deployed at essentially ground level. Such systems are in contrast to single dish observations in which the feed is at an altitude of tens of meters or more. At such altitudes, a feed is susceptible to many low-level, scattered RFI sources, including those from over the horizon, and experience has shown that single dish RRL observations are rarely, if ever, successful during the day, even on days when the general RFI level should be quite low (e.g., the Easter holiday). Dipoles deployed at ground level should suppress RFI sources located near the horizon and should enable more sensitive RRL observations ${ }^{1}$, a hypothesis generally confirmed by daytime observations with the dipole-based UTR-2.

\subsection{Long wavelength array (LWA)}

The Long Wavelength Array (LWA) is intended to be a nextgeneration, high angular resolution imaging telescope operating at frequencies between 20 and $80 \mathrm{MHz}$ (Ellingson et al. 2009). Some of the key science projects are the high redshift Universe, including searches for high-redshift radio galaxies and studies of clusters of galaxies; probing particle acceleration in supernova remnants, radio galaxies, and clusters of galaxies; and searches for radio transients. The telescope would be composed of as many as 50 dipole phased array "stations," with each station containing 256 dual polarization dipoles. The first station (LWA-1) is currently under construction and is planned to conduct science observations in its own right.

The configuration of LWA-1 is a pseudo-random distribution of the dipoles (Kogan \& Cohen 2009), aimed at minimizing the sidelobes from the phased-array station. Because of the broad frequency range, the filling factor of the station is frequency dependent.

Table 4 summarizes our estimates for the required integration times to detect Galactic plane RRLs with LWA-1 over its operational frequency range. We assume $5 \sigma$ detections of roughly $10^{-3}$ optical depth lines at roughly $2 \mathrm{~km} \mathrm{~s}^{-1}$ velocity resolution. We have also assumed an absorber size of $4^{\circ}$, which is comparable to the station resolution at the higher frequencies.

\footnotetext{
${ }^{1}$ Presumably the suppression of RFI should be less important for the Lunar Radio Array (Sect. 5.4).
}

Table 4. Estimates for detecting galactic plane RRLs with LWA-1.

\begin{tabular}{ccccccc}
\hline \hline $\begin{array}{c}v \\
(\mathrm{MHz})\end{array}$ & $n$ & $f$ & $N_{\text {lines }}$ & $\mathrm{DF}$ & $\begin{array}{c}T_{\text {sys }} \\
\left(\times 10^{4} \mathrm{~K}\right)\end{array}$ & $\begin{array}{c}\Delta t_{\text {int }} \\
(\mathrm{h})\end{array}$ \\
\hline 20 & 683 & 0.92 & 10 & 0.26 & 29.5 & 50 \\
25 & 640 & 0.87 & 8 & 0.41 & 18.3 & 28 \\
30 & 603 & 0.76 & 12 & 0.59 & 12.4 & 6 \\
35 & 572 & 0.63 & 10 & 0.8 & 8.9 & 5.5 \\
40 & 548 & 0.5 & 8 & 1 & 6.7 & 7 \\
45 & 526 & 0.4 & 6 & 1 & 5.2 & 15 \\
50 & 508 & 0.33 & 6 & 1 & 4.1 & 23 \\
55 & 492 & 0.27 & 10 & 1 & 3.3 & 10 \\
60 & 478 & 0.23 & 10 & 1 & 2.7 & 14 \\
65 & 466 & 0.2 & 8 & 1 & 2.3 & 24 \\
70 & 454 & 0.17 & 8 & 1 & 1.9 & 32 \\
75 & 444 & 0.15 & 6 & 1 & 1.7 & 56 \\
80 & 434 & 0.13 & 6 & 1 & 1.4 & 72 \\
\hline
\end{tabular}

Notes. $T_{\text {sys }}$ is derived from the GSM sky models (de Oliveira-Costa et al. 2008). It is the average temperature along the Galactic Plane towards Galactic center $\left(-5^{\circ}<b<5^{\circ}, 340^{\circ}<\ell<20^{\circ}\right)$ after convolving the models to LWA-1 beam size and removing the pixels with the highest and lowest $2 \%$ of intensity. The dilution factor, DF, assumes $4^{\circ}$ cloud sizes. The filling factor $f$ is derived using the station dipole layout design (Kogan \& Cohen 2009) and accounts for overlaps and edge effects. $N_{\text {lines }}$ is based on two contiguous bands with roughly $2 \mathrm{~km} \mathrm{~s}^{-1}$ velocity resolution. The integration time to detect lines with optical depths of $10^{-4}$ is $100 \times$ the values listed here.

This first station of the planned LWA instrument is ideal for RRL detection work due to both its flexible frequency coverage and its high filling factor at lower frequencies. At optimum frequencies, the desired sensitivity may be reached in as little as $6 \mathrm{~h}$.

While there has been some consideration of precursor Dark Ages observations with LWA-1, the prime science mission for the LWA as a whole does not include cosmological H I measurements. Moreover, it is not clear that LWA-1 would have the requisite sensitivity to detect the Dark Ages signal. Consequently, RRLs are not likely to be important as a contaminant in any other LWA-1 observations.

\subsection{LOw Frequency ARray (LOFAR)}

The LOw Frequency ARray (LOFAR) ${ }^{2}$ is a next-generation, low radio frequency telescope operating at frequencies between 30 and $240 \mathrm{MHz}$. Its frequency coverage is split between a highband antenna (HBA, 110-240 MHz) and low-band antenna (LBA, [10] 30-90 MHz).

One of its key science projects is to study the Epoch of Reionization using the high-band antenna at redshifts $11.5>z>$ $6.5(115 \mathrm{MHz}<v<190 \mathrm{MHz})$. This is the frequency range over which the RRLs switch from absorption to emission; at some frequencies during this transition they may be undetected (Payne et al. 1989).

In its full configuration, LOFAR has a very low areal filling factor, making it unsuitable for RRL detection and study. However using the "super-station" core of 6 stations within a $300 \mathrm{~m}$ diameter area or using a single station, more reasonable filling factors of order $10 \%$ can be achieved. The instrument has flexible frequency coverage and it is possible to cover large bandwidths (up to $48 \mathrm{MHz}$ ) simultaneously. The ability to fold together many lines will help with detection. However, the

2 http://www.lofar.org 
frequency setup has a fixed frequency resolution, corresponding to about $5 \mathrm{~km} \mathrm{~s}^{-1}$ velocity resolution at the lowest frequencies; thus the narrower RRLs may be under-resolved.

By folding together hundreds of transitions and using the super-station core, this instrument would be able to detect $10^{-3}$ optical depth lines in a few hours at the lowest frequencies; longer integrations should be able to detect these and fainter lines at higher frequencies or with less folding or both. Folding together adjacent RRLs assumes that $\Delta n \ll n$, and thus that the transitions are essentially identical. This assumption is unlikely to be warranted if hundreds of lines are folded together, and the resulting line profile would no longer be useful for deriving physical parameters of the absorbing gas. The planned EoR experiment will integrate deeply enough to detect RRLs, if present, at the higher frequencies.

\subsection{Square Kilometre Array (SKA)}

The Square Kilometre Array (SKA) ${ }^{3}$ will be one of a suite of new, large astrophysics facilities for the 21 st century and will probe fundamental physics, the origin and evolution of the Universe, the structure of the Milky Way Galaxy, and the formation and distribution of planets. Highlighting the SKA Science Case are Key Science Projects (KSPs, Carilli \& Rawlings 2004; Gaensler 2004), one of which is "Probing the Dark Ages and the Epoch of Reionization" (Carilli et al. 2004). The goal is to track the transition of the originally neutral IGM into its current mostly ionized state via imaging of the (highly-redshifted) $\mathrm{H}$ I line. With reference to the three $21-\mathrm{cm}$ cosmology epochs (Sect. 3), the goal for the SKA is to cover the EoR, and as far back into the epoch of first star formation as possible $(20 \lesssim z \lesssim$ 7, $70 \mathrm{MHz} \lesssim v \lesssim 200 \mathrm{MHz}$ ).

The configuration of the low-frequency component of the SKA, the so-called SKA-lo, remains a matter of active study, but the current specifications forsee approximately $25 \%$ of the total collecting area within a diameter of $1 \mathrm{~km}$ and approximately $50 \%$ of the total collecting area within a diameter of $5 \mathrm{~km}$ (Schilizzi et al. 2007). The resulting filling factors are $f \sim 0.5$ $(f \sim 0.05)$ within the central $1 \mathrm{~km}(5 \mathrm{~km})$.

Scaling from the results in Table 4, the central portion of the SKA-lo could therefore detect RRLs along the Galactic plane in integration times of order $5 \mathrm{hr}$. Given the primary scientific driver for the SKA-lo, however, it is unlikely that it will be conducting deep observations along the Galactic plane. Rather, it will likely conduct its deep observations $(\gtrsim 100 \mathrm{~h})$ at high Galactic latitudes. The strength and distribution of RRLs at high Galactic latitudes is currently unstudied; it is unknown if they will be present at all. However, even if the RRLs on these sightlines are an order of magnitude weaker than currently known lines, with $\tau \sim 10^{-4}$, Eq. (2) shows that they would still be detected in integrations of order 100 hours.

\subsection{Lunar Radio Array (LRA)}

The Lunar Radio Array (LRA) is a concept for a radio telescope operating at frequencies around $100 \mathrm{MHz}$ and sited on the far side of the Moon with a prime mission of making precision 21-cm cosmological measurements. With reference to the three 21-cm cosmology epochs (3), the goal for the LRA is to probe at least the epoch of first star formation, and as deeply into the Dark Ages as possible $(100 \lesssim z \lesssim 15,10 \mathrm{MHz} \lesssim v \lesssim 100 \mathrm{MHz})$.

Some of the initial designs for the LRA follow the configuration analysis of Lidz et al. (2008), who advocate a highly

\footnotetext{
${ }^{3}$ http://www. skatelescope.org
}

compact "super core." For such a configuration, the filling factor is large, potentially as large as $f \sim 0.9$. The result would be to reduce the required integration times potentially by an order of magnitude. However, like the SKA-lo, the LRA would most likely target fields well away from the Galactic plane. Similar comments apply to the LRA as to the SKA-lo regarding such observations.

\section{Conclusions}

We have summarized the state of current observations and the potential for future observations of decametric-wavelength radio recombination lines (RRLs, $v \lesssim 200 \mathrm{MHz}$ ). These lines are of interest both because they offer a sensitive and unique probe of cooler, less dense ionized regions throughout the Galaxy than is probed by traditional higher frequency RRL studies, as well as potentially presenting a confusing foreground for redshifted 21-cm cosmology and astrophysical measurements from the Epoch of Reionization (EoR) and Dark Ages.

There are a number of low radio frequency arrays either under construction or in design and development, including the Long Wavelength Array (LWA), the LOw Frequency ARray (LOFAR), the low-frequency component of the Square Kilometre Array (SKA-lo), and the Lunar Radio Array (LRA). All of these arrays will be capable of observing at frequencies below about $200 \mathrm{MHz}$, and they could be used to study RRLs, but the most important design factor for a telescope is the areal filling factor. For the current arrays, LWA-1 has a filling factor that varies with frequency, from nearly $100 \%$ at its lowest frequencies $(\approx 20 \mathrm{MHz})$ to around $10 \%$ at the highest frequencies $(\approx 80 \mathrm{MHz})$; the super-station core of LOFAR ranges from 0.3 to 0.02 over a similar frequency range (its LBA). The estimated integration times are 10 to $100 \mathrm{hr}$ to detect RRLs at optical depths of order $10^{-3}$; in the case of the LOFAR super-station core, these relatively modest integration times are achieved in part by folding potentially hundreds of lines over large bandwidths (up to $48 \mathrm{MHz}$ ). The SKA-lo and the LRA are still in the design phase, but might likely have higher filling factors, potentially larger than $50 \%$, leading to the detection of these lines in $10 \mathrm{~h}$ or less. Further, all of these telescopes offer the possibility of improved performance as a function of frequency, including both improved spectral bandpasses and larger frequency tuning ranges.

RRLs have been studied extensively along the line of sight to the bright radio source Cas A. However, the weakness of the lines and the subsequent need for long integration times and careful data processing to remove even very low level radio frequency interference (RFI) have hampered efforts for the type of systematic, multi-frequency surveys required to probe the physics of the cool, low density ISM throughout the Galaxy. Although not a key science driver for any of these telescopes, systematic surveys for RRLs could be important secondary science to emerge.

From the standpoint of 21-cm studies of the Epoch of Reionization and the Dark Ages, deep observations (>100 h) will likely be conducted with many of these telescopes to search for the highly redshifted $\mathrm{H}$ I signatures, implying sensitivity to much lower optical depths than has heretofore been possible, of order $10^{-4}$. Simple estimates suggest that, if there is RRL-hosting gas at the high Galactic latitudes that will be targeted for the observations, the RRLs could impose spectral fluctuations that are comparable to or exceed the expected H I signatures. One significant mitigating factor, however, is that the lines are likely to be quite narrow, relative to the expected $\mathrm{H}$ I features. $\mathrm{H}$ I signatures from the EoR or Dark Ages are expected to have widths of 
order $0.2 \mathrm{MHz}$ or larger; by constrast, a velocity width of order $10 \mathrm{~km} \mathrm{~s}^{-1}$ near $100 \mathrm{MHz}$ implies a line width of only a few $\mathrm{kHz}$. Thus, the RRLs might likely be narrow, with a low spectral occupancy. The lines might easily be treated as non-terrestrial RFI.

Two final comments are warranted regarding extremely deep observations, particularly as they relate to efforts to detect the $\mathrm{H}$ I signal from the Epoch of Reionization and before. It is not yet known what is the most relevant frequency range for $21-\mathrm{cm}$ cosmology. However, it is possible that LOFAR-HBA and SKA-lo, and possibly the future LRA, would be observing in the regime for which RRLs transition from emission at higher frequencies to absorption at lower frequencies. In this case, the lines would not be detected. Second, most of our comments have concerned $\alpha$-transition lines. In deep integrations, $\beta$-, $\gamma$-, and potentially $\delta$-transition lines can be detected (Stepkin et al. 2007). The resulting spectral occupancy would be much higher and the ability to fold or remove the lines could be reduced substantially.

Acknowledgements. The authors thank E. Polisensky for providing sky temperatures, A. Cohen for help with calculating the station filling factor for the LWA-1, G. Smirnov for information about previous instruments, and the referee for suggestions that helped us clarify and quantify certain points. This research has made use of NASA's Astrophysics Data System. The LUNAR consortium is funded by the NASA Lunar Science Institute (via Cooperative Agreement NNA09DB30A) to investigate concepts for astrophysical observatories on the Moon. Basic research in radio astronomy at the Naval Research Laboratory is funded by 6.1 Base funding.

\section{References}

Anantharamaiah, K. R., Payne, H. E., \& Erickson, W. C. 1988, MNRAS, 235, 151

Blake, D. H., Crutcher, R. M., \& Watson, W. D. 1980, Nature, 287, 707
Bridle, A. H. 1967, MNRAS, 136, 219

Carilli, C. L., Furlanetto, S., Briggs, F., et al. 2004, in Science with the Square Kilometre Array, ed. C. L. Carilli, \& S. Rawlings (Amsterdam: Elsevier), 1029

Carilli, C. L., \& Rawlings, S. 2004, Science with the Square Kilometre Array (Amsterdam: Elsevier), New Astron. Rev.

Ellingson, S., Clarke, T. E., Cohen, A., et al. 2009, Proc. IEEE, 97, 1421

Erickson, W. C., McConnell, D., \& Anantharamaiah, K. R. 1995, ApJ, 454, 125

Furlanetto, S. R., Oh, S. P., \& Briggs, F. H. 2006a, Phys. Rep., 433, 181

Furlanetto, S. R., Oh, S. P., Pierpaoli, E. 2006b, Phys. Rev. D, 74, 103502

Gaensler, B. M. 2004, Key Science Projects for the SKA SKA Memorandum 44, http://www. skatelescope.org

Golynkin, A. A., \& Konovalenko, A. A. 1991a, SvA Lett., 17, 7

Golynkin, A. A., \& Konovalenko, A. A. 1991b, SvA Lett., 17, 10

Gordon, M. A., \& Sorochenko, R. L. 2002, Radio Recombination Lines: Their Physics and Astronomical Applications (Kluwer: Dordrecht)

Harker, G., Zaroubi, S., Bernardi, G., et al. 2009, MNRAS, 397, 1138

Helmboldt, J., \& Kassim, N. E. 2009, AJ, 138, 838

Kantharia, N. G., \& Anantharamaiah, K. R. 2001, J. Astrophys. Astron., 22, 51

Kantharia, N. G., Anantharamaiah, K. R., \& Payne, H. E. 1998, ApJ, 506, 758

Kogan, L., \& Cohen, A. 2009, LWA Memorandum \#150; http://www.ece. vt.edu/swe/lwa/lwa0150.pdf

Konovalenko, A. A. 1984, SvA Lett., 10, 384

Konovalenko, A. A., \& Sodin, L. G. 1980, Nature, 283, 360

Lidz, A., Zahn, O., McQuinn, M., Zaldarriaga, M., \& Hernquist, L. 2008, ApJ, 680,962

de Oliveira-Costa, A., Tegmark, M., Gaensler, B. M., et al. 2008, MNRAS, 388 , 247

Payne, H. E., Anantharamaiah, K. R., \& Erickson, W. C. 1989, ApJ, 341, 890

Payne, H. E., Anantharamaiah, K. R., \& Erickson, W. C. 1994, ApJ, 430, 690

Pritchard, J. R., \& Loeb, A. 2008, Phys. Rev. D, 78, 103511

Schilizzi, R. T., et al. 2007, Preliminary Specifications for the Square Kilometre Array, SKA Memorandum 100, http://www.skatelescope.org/PDF/ memos/100_Memo_Schilizzi.pdf

Sorochenko, R. L., \& Smirnov, G. T. 1990 Radio Recombination Lines: 25 Years of Investigation, IAU Colloq. 125, 163, 189

Stepkin, S. V., Konovalenko, A. A., Kantharia, N. G., \& Udaya Shankar, N. 2007, MNRAS, 374, 852 